\title{
A Combustible/Electronegative Gas Detector Utilizing Uranium Doped Cast Ceramic Microchannels
}

\author{
John D. Olivier and Chester G. Wilson \\ Institute for Micromanufacturing, Louisiana Tech. University
}

\begin{abstract}
This paper reports on an on-chip radioactive microdevice constructed from a lost mold ceramic casting process. Uranium acetate nanoparticles are imbedded into this room temperature curing ceramic process. This cast microchannel provides a robust, on chip beta particle (fast electron) source, which is used for electronegative gas collection, and gas mobility determination. These are both standard macroscale techniques for combustible gas identification. It can either serve to ionize the local gas, which is being measured, or it can be captured by local electronegative gas. The beta source is biased to ground, and a separate current collection electrode is biased positively or negatively. This measures local ion/electron generation, and provides mobility information. As these mobilities and ionization constants are unique to particular gas species, different gas conditions provide different current collection signatures. Air, helium, alcohol and carbon dioxide are characterized with this device.
\end{abstract}

\section{INTRODUCTION}

Explosive gas detection is important to security and industrial applications. Tin-oxide gas detectors are probably the most common combustible gas detector; the measured gas interacts with absorbed oxygen, resulting in a conductivity change. While array-based detectors of this type have improved performance dramatically, these devices have limitations in determining the exact type of gas; both propane and carbon dioxide result in a resitivity change $[1,2]$. Several micro-gas chromatographs have been developed, but these are highly complex devices, and fabrication is costly $[3,4]$. Small scale plasma spectroscopic devices have been developed, but these devices require hundreds of volts to operate. $[6,7]$. Several small scale devices that rely on ionmobility measurements have also been developed, and would further be improved with the use of tunable onchip radioactive sources [8,9]. Electroplated nickel isotope microdevices have been constructed for on- chip power sources, but not for gas detection [10]. Electronegative gas absorption is a common technique in analytical chemistry; this uses a large low energy beta particle emitting source, which ionizes a carrier gas, and certain gas types absorb electrons, changing measured current.

The technology described here allows: 1) a wide variety of radioactive materials that could not be electroplated to be cast in ceramic at room temperature, 2) high energy particle sources that serve to ionize both carrier gasses and the gas to be measured, and 3) on chip electrodes that can collect the created ions and electrons, which are characteristic of the measured gas (Fig. 1).

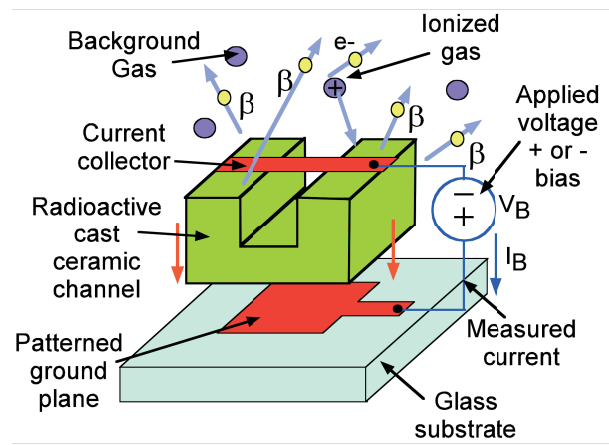

Fig. 1 Uranium particles embedded in the ceramic micro-channel emit high energy beta partcles, which ionize proximal gasses. The ionization constant and mobilities can be measured using patterned electrodes.

\section{RADIOACTIVE MICROCASTINGS}

The uranium-doped ceramic microcastings are manufactured with a room temperature lost-mold process (Fig. 2). The undoped ceramic castings are created using a two part, dry and wet, compound. The first component is an alumina ceramic powder, and the wet activator is a nanoparticle colloid.

The inexpensive fabrication process allows an onchip $0.543 \mathrm{MeV}$ beta particle source (Fig. 3). A uranium-doped microchannel is fabricated, and then imbedded into a microsandblasted glass cavity, where electrodes have been patterned (Fig. 4). This high- 
energy source liberates secondary electrons in the ceramic, and it either ionizes or it is captured by the background gas. The source activity can be tailored by changing the dopant levels (Fig. 5). Several mm thick polymer coatings only attenuate about $50 \%$ of the beta emission, so the source can be encapsulated (Fig. 6). As the radioactive castings are small, the emitted flux falls off quickly with distance, mitigating safety problems (Fig. 7).

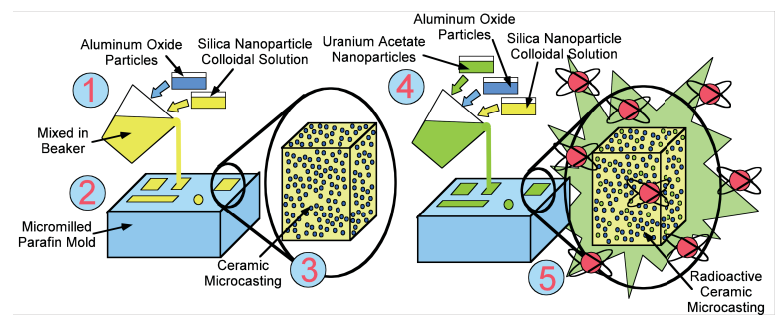

Fig. 2 Using room temperature curing ceramics composed of aluminum oxide particles with a silica nanoparticle colloidal activator (1) Micro-castings of varying geometries are created (2). This solution hardens to a $10^{9} \mathrm{ohm}-\mathrm{cm}$ ceramic at room temp. (3). When uranium particles are added (4), an on-chip radioactive source is created (5). Flux and radioactive loading can be tuned.

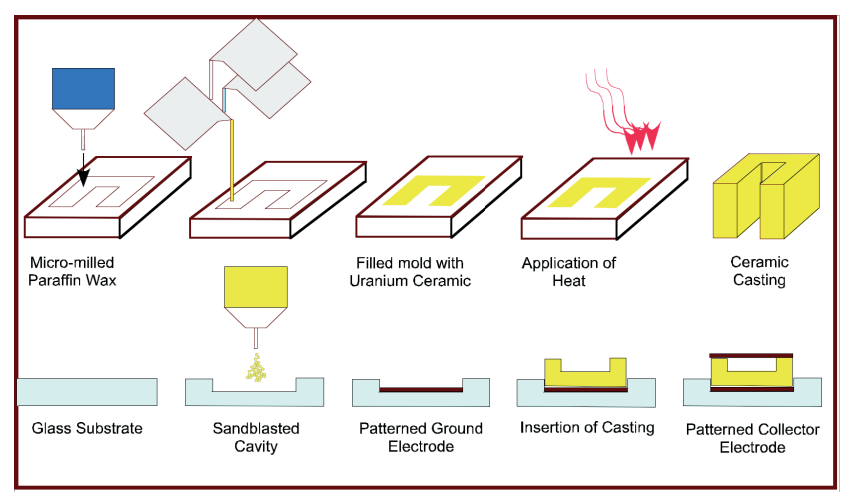

Fig. 3 Ceramic radioactive castings are made with a lost wax process, these castings are imbedded into a cavity sandblasted in glass, with a patterned ground plane and collector. An optional polymer coating can be applied over ceramic casting to protect the source.

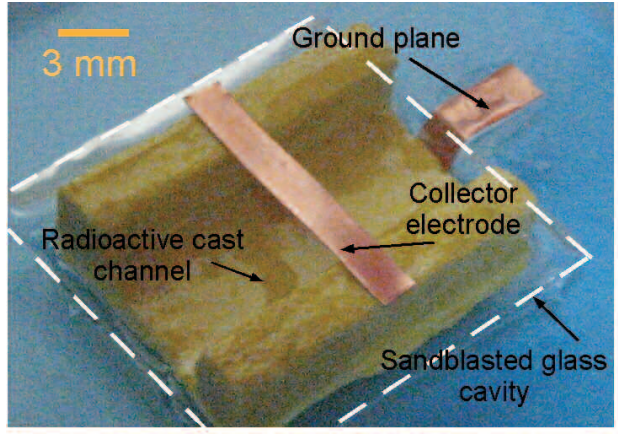

Fig. 4 Uranium microdevice is yellow, and imbedded in microsandblasted glass cavity.

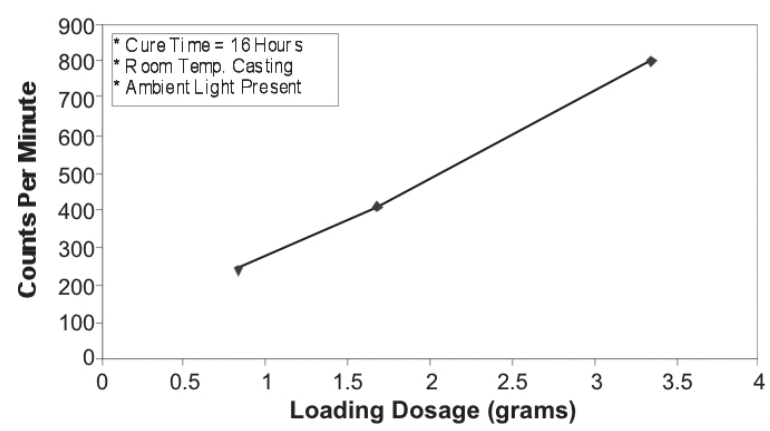

Fig. 5 Radioactivity, and particle flux is linear with uranium loading into ceramic. Counts per minute are higher than that caused by beta emission of uranium alone; secondary emissions are produced from aluminum oxide particles.

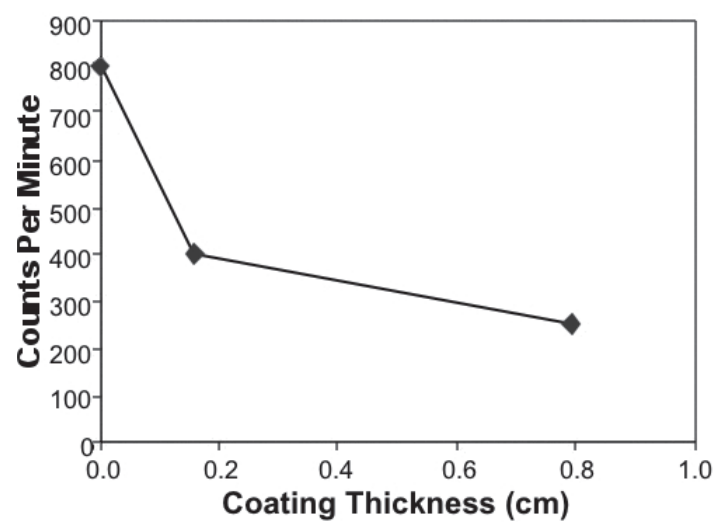

Fig. 6 Coating the nuclear ceramic material with polymer films causes a minor decrease in beta emission. This is desirable because emission can be finely tuned using very inexpensive methods. 


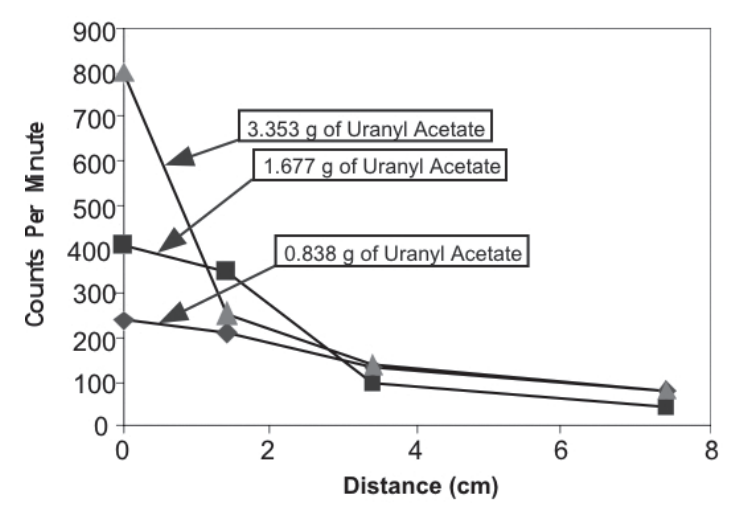

Fig. 7 As the distance of three distinctively doped ceramic micro-castings increases from a beta detector, the counts emitted per minute of the micro-casting falls off very rapidly. This results in only locally ionized gas, and increases safety.

\section{EXPERIMENTAL RESULTS}

To characterize these devices, initial testing was done in dried air, where the biasing electrode is fed into a current collecting $38 \mathrm{pF}$ ceramic capacitor (Fig. 8). Current collection in air is substantially increased above background with the addition of the nuclear source, allowing larger charge capture (Fig. 9). As the background atmosphere is heated, current collection is considerably increased, as moisture is reduced, and ionization probabilities increased. When the biasing electrode is positive, electrons are captured, along with negative ions. Negative biasing collects positive ions, due to the large difference in mobilities, the currents of the two species vary several fold (Fig. 10). Increasing the collector bias voltage increases the species currents differently, until they become equal at saturation (Fig. 11). The current collected is insensitive to electrode area; several fold increases in capture area result in only incremental increases in current (Fig. 12). Helium, a frequent carrier gas was tested; noble gases do not capture electrons, and the ion/electron mobility difference is found considerably higher (Fig. 13). With helium, electron current collection occurs much faster than with less mobile ion collection.

This device was tested with alcohol vapors and in the presence of a carbon dioxide ambient. Carbon dioxide, an electronegative gas which captures electrons, provides a greater current when the electrode is biased positively (Fig. 14).

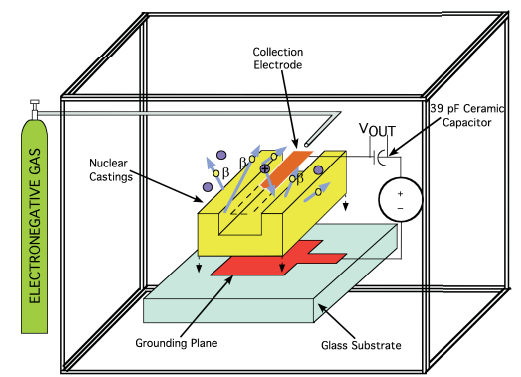

Fig. 8 Testing was implemented in a controlled chamber. Charge is collected on a ceramic capacitor, over seconds, resulting in $\mathrm{mV}$ signals.

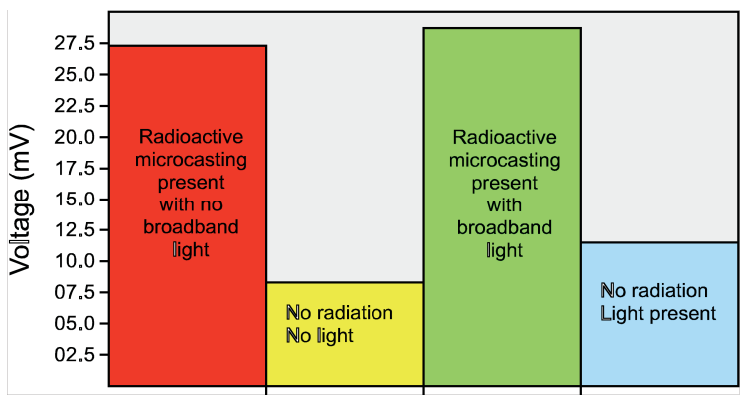

Fig. 9 The radioactive source increases ionization of background gas several-fold.

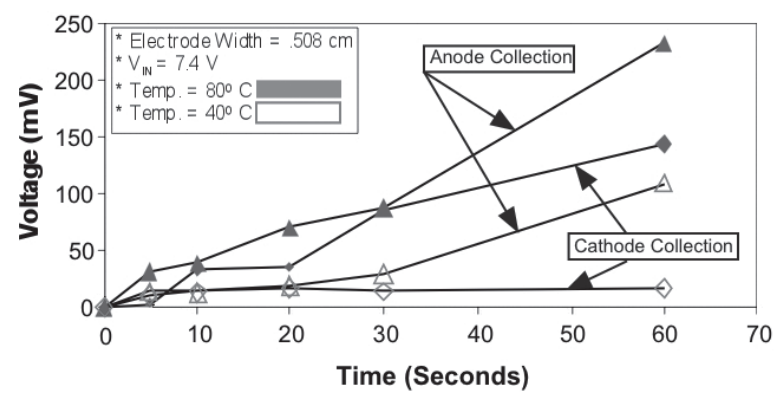

Fig. 10 Elevated temperatures substantially increase collected currents in air.

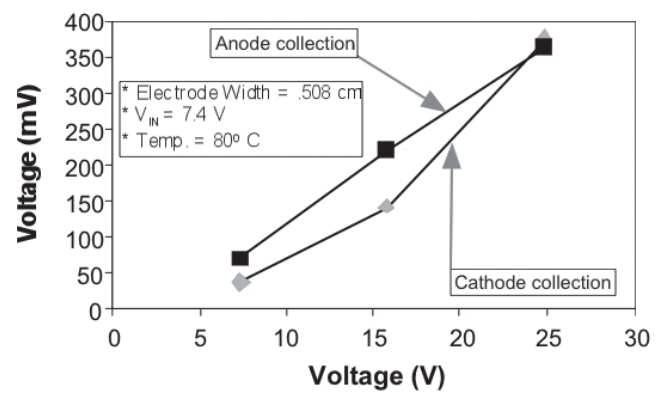

Fig. 11 As the collector bias is increased, anode and cathode current increases, until they match. 


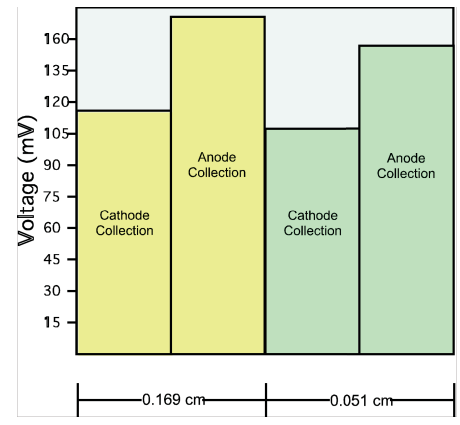

Fig. 12 Anode and cathode current collection is fairly insensitive to electrode sizes. As electrode size decreases little signal attenuation is observed for both cathode and anode collection.

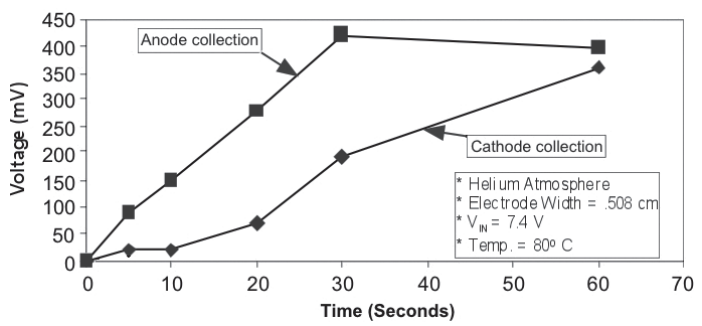

Fig. 13: Helium, a noble gas with a small ionization potential captures no electrons, so larger anode currents are seen initially. After sufficient time, collection currents become equal.

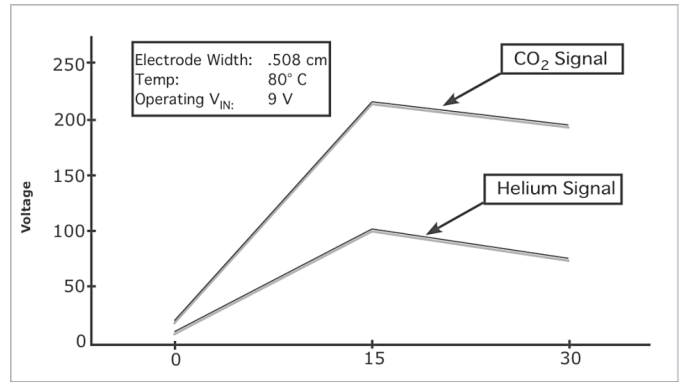

Fig. 14: Carbon dioxide, which captures electrons, provides higher anode current than alcohol vapors.

\section{CONCLUSIONS}

A new fabrication technique to produce uranium doped ceramic microcastings is presented. Unique to the fabrication process is the ability to controllably dope the ceramic castings with varying types of radiating nuclear isotopes. The device presented here utilizes a cast ceramic microchannel doped with uranyl acetate, which provides an ionization source that has been tested for use in gas mobility measurements. An on-chip electrode serves to collect positive/negative ions and electrons. Beta emission from these sources serves a two-part role in the device functionality, they ionize the background gas to be tested, and they provide a source of electrons that are captured by the gas. The device has been characterized in air, helium, alcohol vapors, and carbon dioxide, resulting in different voltage-current characteristics.

\section{ACKNOWLEDGEMENTS}

This material is based upon work in part supported by the DARPA and SPAWAR SC under award \# N66001-051-8903. This work was also funded in part by the Louisiana Board of Regents Research Competitiveness Subprogram.

\section{REFERENCES}

[1] R. Cavicchi, et. al., "Microhotplate Gas Sensor Arrays", Proc. of SPIE, 1999, pp. 38-49

[2] B. Panchapakesan, D.L. Devoe, R.E. Cavicchi, R.M. Walton, and S. Semancik, "Micromachined Array Studies of Tin Oxide Films: Nucleation, Structure, and Gas Sensing Characteristics" MRS Symposium Proc,, 574, 1999, pp. 213218

[3] L.C. Chia-Jung, J. Whiting, R.D. Sacks, and E.T. Zellers, "Portable Gas Chromatograph with Tunable Retention and Sensor Array Detection for Determination of Complex Vapor Mixtures," Analytical Chemistry, (75)6, Mar. 2003, pp. 1400-1409

[4] Y. Kawamura, S. Konishi, M. Nishi, "Development of a Micro Gas Chromatograph for the Analysis of Hydrogen Isotope Gas Mixtures in the Fusion Fuel Cycle," Fusion Engineering and Design, v58-59, Nov 2001, pp. 389-394

[6] J.C.T. Eijkel, H. Stoeri, A. Manz, "A dc microplasma on a chip employed as an optical emission detector for gas chromatography," Anal. Chem. 72, 6/00, pp. 2547-2552

[7] C. G. Wilson, Y. B. Gianchandani, "LEd-SpEC: Spectroscopic detection of water contaminants using glow discharge from liquid electrodes," IEEE Transactions on Electron Devices, 49/12, Dec 2002, pp. 2317-2322

[8] R. A. Miller, E. G. Nazarov, G. A. Eiceman, and A. King, "A MEMS radi-frequency ion mobility spectrometer for chemical vapor detection," Sensors and Actuators, (91)3, 2001, pp. 301-312

[9] W. Chambers, J. Phelan, et. al., "Explosive ordinance detection in land and water environments with solid phase extraction/ion mobility spectrometry," Proc. of SPIE, v 3710, n I, 1999, pp 290-298

[10] H. Li, A. Lal, et. al., "Self-Reciprocating RadioisotopePowered Cantilever”, Jour. Appl. Phys., 2001, pp. 1122. 